\title{
Variations
}

Variations

Revue internationale de théorie critique

$16 \mid 2012$

Tahrir is here !

\section{Critique de l'État : la constellation négative}

Pierre Clastres, Claude Lefort, Miguel Abensour et Françoise Proust

\section{Lucia Sagradini}

\section{(2) OpenEdition}

Journals

\section{Édition électronique}

URL : http://journals.openedition.org/variations/202

DOI : 10.4000 /variations.202

ISSN : 1968-3960

Éditeur

Les amis de Variations

Édition imprimée

Date de publication : 16 janvier 2012

\section{Référence électronique}

Lucia Sagradini, «Critique de l'État : la constellation négative », Variations [En ligne], 16 | 2012, mis en ligne le 20 février 2012, consulté le 02 mai 2019. URL : http://journals.openedition.org/variations/202 ; DOI : 10.4000/variations.202

Ce document a été généré automatiquement le 2 mai 2019.

Les ami•e•s de Variations 


\title{
Critique de l'État : la constellation négative
}

\author{
Pierre Clastres, Claude Lefort, Miguel Abensour et Françoise Proust
}

Lucia Sagradini

\section{NOTE DE L'ÉDITEUR}

Première publication sur www.theoriecritique.com, « Tahrir is here ! ", 16 janvier 2012, pp. 56-64

1 Il y a toujours un début à tout. Aujourd'hui, d'une certaine manière, nous pourrions dire que depuis 2001, et la crise politique qu'a vécue l'Argentine, nous sommes entrés de plein fouet dans le XXIe siècle. Ainsi ce texte se propose, avec audace, non pas seulement de mettre en lien et en perspective les différents événements qui traversent une société, ni même un continent, mais bien de relier les différentes crises qui marquent le surgissement d'un temps nouveau: celui de l'agir démocratique revivifié contre les formes réifiées des États.

2 La situation globale est telle, elle apparaît dans une séquence historique d'une telle intensité, qu'il devient impossible de ne pas mettre en lien ce que les médias voient et transmettent sans saisir les enjeux démocratiques profonds qui traversent les événements, impossible de s'en tenir à leurs singularités et de ne pas oser l'audace d'un rapprochement conceptuel. La proposition est alors dans cet article de tirer parti du scintillement d'une constellation négative d'auteur-e-s qui a eu l'audace de tenir et de porter la critique d'une coïncidence de l'État et de la démocratie pour dégager l'écart et la tension réelle qui existent entre démocratie, appareil de coercition et régulation ${ }^{1}$.

Les événements qui ouvrent sur ce nouveau siècle marquent l'impossibilité de mépriser cette non-coïncidence et de réaliser son occultation par la mise en place d'un discours dominant où souvent l'État se superpose à la politique, mais aussi, autre versant, où la démocratie est enferrée dans le capitalisme. Les pratiques politiques actuelles qui ici renversent les régimes (Maghreb), là les gouvernements (Islande), ailleurs refusent la 
toute puissance du FMI (Grèce) ou qui contestent l'économie de marché (USA), ces pratiques politiques nous indiquent dans la vivacité de l'action combien l'écart est grand et combien il est en train de se creuser.

4 En effet, les expériences qui agitent le monde un peu partout affrontent et mettent en brèche le système qui s'impose à nous: le capitalisme et ses formes nouvelles, plus violentes encore et qui tendent au désastre, comme le dénonce Naomi Klein dans La stratégie du choc, la montée d'un capitalisme du désastre ${ }^{2}$. Dans le même temps où les êtres se battent et se débattent, les discussions entre Nicolas Sarkozy et Angela Merkel, qui polarisent les médias, ne cherchent qu'à confirmer une unique ligne politique: imposer l'austérité, la privatisation des dettes, sous couvert de discours de la raison. Ce discours signale si justement qu'à son revers se tient un vœu déraisonné: celui de l'irrationnel, de privilégier le désastre économique et social pour le plus grand nombre au détriment du bien vivre ensemble. Situation qui provoque aussi des relents abjects de germanophobie dans l'espace public français.

\section{La démocratie contre l'État}

5 Ainsi, si les événements qui occupent et partagent le devant de la scène ont la singularité de leur contexte particulier et sont marqués par des maux ou des difficultés propres, pourtant ils annoncent tous ce désir de changer le monde tel qu'il est. Ne pas écarter ce lien qui les parcourt et permet aussi de les lier a une portée. Cette mise en commun, malgré tout, de ce qui se déroule en Europe, en Asie, au Maghreb, en Amérique est une possibilité pour nous de faire surgir une tension, celle de l'agir démocratique qui a pour caractéristique de se réaliser contre les formes étatiques.

6 Miguel Abensour, dans La démocratie contre l'État, en reprenant le cheminement de Marx dans Critique du droit politique hégélien de 1843 nous ouvre la voie pour reformuler ce qui participe pleinement de la politique. Un agir démocratique contre l'État 3. «Ce faisant, ce que Marx donne à comprendre, dans le dialogue qu'il entretient avec Hegel, Moses Hess et Spinoza, c'est une pensée du politique en son autonomie - hors de toute prétention à le dissoudre dans une genèse empirique -; une pensée du rapport insuperposable entre l'État et la démocratie et d'un jeu permanent d'inadéquation; une pensée affirmative de la souveraineté qui n'est pas seulement d'essence démocratique, quelle que soit la forme d'État, mais qui trouve dans la démocratie comme telle son rapport même d'auto-institution continuée " (Abensour, p.114). Les expériences auxquelles nous assistons manifestent cet enjeu essentiel de réaffirmer ce qu'est la démocratie : un agir contre.

7 Depuis la crise politique que l'Argentine a rencontré suite au crash économique de 2001 et jusqu'aux révoltes qui fleurissent un peu partout en Europe et en Amérique, en passant par la rupture profonde des Révolutions au Maghreb, et l'expression de tensions en Asie, les potentialités et les singularités des pratiques démocratiques se dévoilent au grand jour en même temps que ce capitalisme, vampire au cœur assoiffé, se dénude devant nos yeux hallucinés ou atterrés ${ }^{4}$.

8 Face à l'épreuve de la réalité, la philosophie des années passées et le discours dominant, qui a toujours été au service du système capitaliste, deviennent caduques. Il devient désormais impossible de servir cette potion fantasmatique qui faisait coïncider la démocratie et l'État et, par extension, le marché et la démocratie. Le temps a sonné pour 
les Marcel Gauchet et consorts. Aujourd'hui, saisir le réel à l'œuvre, capter les expériences des gens, implique de reformuler cette idée que Marx avait dégagée, une critique radicale de l'État qui se concentre dans le souhait de voir le dépérissement de l'État ainsi que la réflexion portée à la forme démocratique ${ }^{5}$. Il est pourtant impossible d'écarter le fait que les moments de renversement des formes étatiques sont aussi des temps de dangers. Rien ne peut garantir l'essor d'une forme qui cristallise le dépassement, cependant l'absence d'expériences démocratiques entrave profondément la transformation politique vers la démocratie. Toute la politique guerrière des USA soutenue par l'Union Européenne, le démontre : la démocratie ne s'impose pas aux autres en l'exportant par la violence. Cette grande réserve ne doit pas nous empêcher de problématiser la critique de l'État, mais plutôt la complexifier.

\section{La société contre l'État}

9 Dans La société contre l'État, Pierre Clastres a eu le courage intellectuel, dans une période réifiée, de soutenir cette idée fondamentale que la politique ne coïncidait pas avec l'État. "Même dans les sociétés où l'institution politique est absente (par exemple, où il n'existe pas de chefs), même là le politique est présent, même là se pose la question du pouvoir : non au sens trompeur qui inciterait à vouloir rendre compte d'une absence impossible, mais au contraire au sens où, mystérieusement peut-être, quelque chose existe dans l'absence. Si le pouvoir politique n'est pas une nécessité inhérente à la nature humaine, c'est-à-dire à l'homme comme être naturel (et là Nietzsche se trompe), en revanche il est une nécessité inhérente à la vie sociale. On peut penser le politique sans la violence, on ne peut penser le social sans le politique : en d'autres termes, il n'y a pas de sociétés sans pouvoir ». La possibilité conceptuelle que cet auteur a ouverte en décrivant que l'absence d'État n'était pas une absence de politique, a été un tremblement de terre pour la sphère spécialisée qui s'occupait de la question politique. Bien que cette révolution copernicienne ait eu lieu dans le champ de l'anthropologie et dans l'étude de sociétés amérindiennes, il n'en restait pas moins que Clastres opérait un véritable bouleversement théorique : il était désormais manifeste que le sans État n'était pas absence de politique, mais bien plus une politique contre le surgissement de l'État.

Il réalisait alors un changement de perspective radicale en pensant que "L'histoire des peuples dans l'histoire, c'est l'histoire de leur lutte contre l'État ${ }^{6}$. Cette pensée ouvrait alors des pistes insoupçonnées et vivifiantes qui résonnent chez un Lefort qui conceptualise alors la démocratie comme espace vide ${ }^{7}$ ou un Abensour qui la poursuit dans l'extension de la démocratie contre l'État et qui permet de relire Marx avec un nouvel entrain. La question est alors de dégager les lignes d'horizon d'un courant qui déplace la question de l'économie vers la politique et celle de la fin de l'histoire vers un univers instable où la surprise, l'événement est toujours en mesure de surgir. Le conflit n'est plus liquidé, au contraire il tient la place principale, mais dans une aspiration autre que celle de trouver la forme adéquate ou non de l'État. Au contraire, cette constellation de penseur-e-s politiques tendent à inventer un univers sans arcanes. Un monde de l'auto-institution. Bien sûr, Lefort n'ira pas jusque là, prisonnier de la nécessité de lutter contre les formes du totalitarisme, sa pensée aussi fulgurante que précieuse s'arrête en chemin.

11 Plus avant, Rancière, à son tour, a suivi cette piste, par exemple, lorsqu'il écrit La haine de la démocratie ${ }^{8}$. Il reprend ainsi la tradition venue de cette constellation d'auteur-e-s qui 
passe par la négativité et déplace la question démocratique de la forme réifiée de l'État. Il fait le choix des gens contre la structure.

Aujourd'hui, dans cette accélération vers cette forme mutée de néo-capitalisme dont parle Naomi Klein, se déploient, dans la même séquence historique, agir démocratique globalisé et fuite en avant des États, sous couvert de discours de vertu économique. L'intensité avec laquelle aujourd'hui cette nécessité de penser contre l'État est d'autant plus vitale que l'accélération dans la déliaison et l'anéantissement du bien vivre s'abat si férocement sur les êtres partout dans le monde. Ce nouveau temps, que nous traversons, et qui conjugue critique de l'État et déploiement du néo-capitalisme, dévoile l'abandon du bien vivre par les formes étatiques contemporaines. Il est désormais temps de réaliser aussi la critique d'une démocratie molle qui au fond accompagne le renoncement de l'État à protéger les êtres au profit d'une exploitation dure. Les paliers en sont : l'abandon de la santé, de l'éducation, du bien vivre (retraite et temps de travail) au profit d'une société aux inégalités et injustices accrues.

Partout dans le monde, le rejet de l'État est au cœur des révoltes, luttes et manifestations, malgré leurs différences. Pour évoquer les événements qui ont lieu, John Holloway utilise l'image d'individus au bord d'un lac gelé qui jettent des petits cailloux de chaque côté et provoquent des brèches qui peu à peu vont se rencontrer pour provoquer le craquement de cette couche rigide de glace : le crack capitalism ${ }^{9}$. La mise en lien dans cette image de la brisure du capitalisme est une étape importante pour appréhender la démocratie en dehors des définitions convenues qui en réalité véhiculent la soumission des intellectuels au pouvoir et au monde réifié. Raison pour laquelle Le Monde pouvait se permettre de titrer sur «le silence des intellectuels » face au printemps arabe. En ce sens, les courants marxistes ont aussi véhiculé des contresens sur le rapport de l'État à la démocratie. Mettre en lien ce qui diffère est ainsi un moyen pour sortir d'un univers conceptuel réifié qui ne fait que nous soumettre à un ordre des choses aussi cruel qu'inique et dans lequel l'individu n'a que peu d'intérêt.

En parallèle, rendre compte et problématiser un héritage et un travail conceptuel, qui traverse le temps et qui a pour particularité de s'inscrire dans la négativité, est aujourd'hui non pas une coquetterie, mais une nécessité pour accompagner les subjectivités dans leurs aspirations. Cette constellation, ciel étoilé offert aux spectateurs, porte la capacité de réaliser la critique de l'État et de donner à l'invention, à l'action de concert et à la créativité toutes les potentialités pour penser une société autre. Les traits essentiels de la démocratie portent alors plus d'intérêt que la forme somme toute relativement aboutie de l'État même démocratique pour lier les êtres, cette autre manière de regarder la politique existe et a existé : en dehors de l'État.

\section{Résister avec Françoise Proust}

Dans ce sens, la brèche que Stéphane Hessel a ouvert avec son pamphlet, Indignez-vous !, devenu best-seller, illustre l'articulation entre macro et micro politique ${ }^{10}$. Il semble avoir pour entreprise simple de répondre à la question nodale : que faire face à l'iniquité du monde? La réponse tient en un mot presque, indignez-vous! Le titre de l'ouvrage est aussi sa substance vitale et c'est sans doute la raison pour laquelle il a tellement retenu l'attention. Car tel un sésame, la parole d'Hessel, Ali Baba de nos récits modernes, trouve une formule magique qui contient la sève essentielle de la pulsation politique. Un moteur révélé par la force de parole qu'Hessel a su canaliser et qui révèle aussi les étendues 
parfois oubliées du langage et de l'adresse à l'autre. Par le verbe, l'auteur rappelle que la ressource ultime des êtres face à l'injustice, sous toutes ses formes, se trouve dans un sentiment négatif, l'indignation, et derrière lui, la colère. C'est dans ce sentiment que l'individu peut trouver les ressources internes pour agir et réagir. Il s'agit ici d'une mise en forme qui reprend le travail poussé en profondeur par Françoise Proust dans son ouvrage, De la résistance ${ }^{11}$. Elle réalise un travail de fond afin de nouer le fil qui lie la politique et la résistance. La finesse du propos n'en est que plus lumineuse quand nous nous souvenons que les révoltes des jeunes (2005) en France ont surgi à la suite de la mort de deux adolescents, Zyed Benna et Bouna Traoré, à Clichy-sous-bois. Mais aussi qu'en Tunisie (et par extension au Maghreb) c'est l'immolation d'un jeune homme, Mohamed Bouazizi, désespéré de ne pouvoir travailler - en vendant des fruits pour vivre - qui a été l'événement déclencheur des Révolutions.

Ce sentiment de colère face à l'injustice manifeste aussi la capacité d'empathie des êtres au cœur de la politique. Il montre combien les êtres ne sont pas indifférents au sort des autres et que, parfois, face à l'inacceptable, ils peuvent trouver les ressorts internes pour rejeter l'injustice et l'État qui ne protège plus, mais s'abat sur les êtres. Aujourd'hui, partout dans le monde, quand l'État n'accomplit plus son rôle de protéger le vivreensemble, il se trouve confronté à la colère salutaire des êtres et à leurs aspirations à un mieux vivre.

Une des limites de la situation est de voir par exemple une forme religieuse d'État venir supplanter les formes autoritaires connues. Le fait que la religion porte en elle la promesse d'une utopie est en ce sens fortement problématique. L'exemple iranien nous a déjà dévoilé combien, dans la réalité, il s'agit d'une non-rencontre entre utopie et État religieux. Pour autant, dans le magma et l'effervescence politique, la possibilité de basculer du mauvais côté est bien réelle. Mais, quelle que soit la suite de la séquence historique a laquelle nous assistons, il est indispensable de retenir l'étincelle de l'instant qui porte en lui tant des promesses.

\section{Les trois mamelles de la démocratie : agir, bien vivre, s'auto-instituer}

De manière certaine, cette séquence historique rapproche des manifestations, des soulèvements et des Révolutions, que la science politique et les catégories scientifiques tiennent en général écartés. Comme l'a souligné Naomi Klein : nous avons changé de paradigme, ces événements manifestent surtout la présence d'un agir démocratique, impossible à contourner, l'agir contre les formes étatiques. Vif, en ordre de combat, prêt à relever le défi d'une forme politique qui, si elle s'impose comme forme rationnelle (et indépassable), connaît pour autant de sérieuses difficultés. Car la violence déguisée, plus ou moins voilée, des formes étatiques n'est plus aujourd'hui que la « police » d'un marché -travail abstrait - ne cesse de s'éloigner du réel pour assouvir une pulsion aussi déraisonnée que morbide (destruction des êtres et du vivant), ne laisse aux individus que le squelette décharné d'une société où l'iniquité et la soumission se projettent dans l'asservissement $\mathrm{du}$ plus grand nombre au pouvoir financier. La violence des formes étatiques et la nature même de l'État sont aujourd'hui tournées vers la poursuite et la réalisation d'un monde où les êtres sont asservis aux lois d'un capitalisme toujours plus abstrait. 
19 Il est alors heureux de lire Clastres et de regarder la réalité pour se rappeler cet écart entre démocratie et État. Un savoir que l'expérience de ce début de XXIe siècle nous dévoile malgré toutes les tentatives d'occultation. Les expériences vécues, ici, là-bas, à Moscou, à Londres, ou Athènes, nous le crient et nous obligent à les considérer dans leur qualité d'agir contre. Comme la Révolution française le fit avec Hegel, Heine ou Marx, aujourd'hui, nous devons pour le moins être à la hauteur, en tant que spectateurs actifs ; à nous de saisir les enjeux conceptuels de ces Révolutions, de ces révoltes, soulèvements, émeutes auxquels nous assistons depuis 2001 et qui ont aussi renversé la géopolitique du centre et de la périphérie. Brouillant les pistes d'un monde organisé d'un haut vers le bas, les lignes et les plaques tectoniques ont bougé et le monde a changé, même s'il bougera encore, à nous de l'enregistrer. Le point essentiel est d'assimiler cet écart de la démocratie en train de se faire, et qui offre la promesse d'un véritable coup d'arrêt à la violence d'un ordre établi qui craque.

Le second point qui caractérise ces événements et la démocratie qu'ils manifestent est qu'il s'agit de remettre la question du bien vivre au cœur de la politique. Il se trouve que cet écart entre la démocratie et l'État dont Abensour signale le travail est aussi une tension qui peut dans un premier temps réaliser un rappel à l'ordre de la forme réifiée de l'État. En effet, l'auteur nous rappelle que si l'État s'éloigne trop de ses origines, à savoir la fondation révolutionnaire et les aspirations des êtres, alors la démocratie en tant qu'expression de l'agir des individus peut venir peser sur l'État pour imposer une reprise en compte des aspirations qui sont à la base de sa formation - une réorganisation du social tournée vers le bien vivre, "parce que l'État représente pour la démocratie un danger permanent de dégénérescence », (Abensour, p. 108).

Lorsque l'État s'éloigne trop fortement et se retourne contre les êtres, alors, il connaît le risque de se voir contester et bouleverser par l'agir démocratique. L'agir démocratique est alors démocratie sauvage comme le souligne Abensour. La démocratie sauvage s'offre alors soit comme limite qui s'impose à l'État et peut se considérer comme un élément régulateur qui vient porter un coup d'arrêt à l'entreprise de la forme étatique, et en même temps peut alors aussi la protéger d'elle-même. Mais elle peut aussi être la source $\mathrm{du}$ changement structurel par l'invention de formes nouvelles, mieux adaptées aux souhaits des êtres.

Non seulement, l'aspiration démocratique est une manifestation contre l'inique. Mais chaque événement singulier de contestation manifeste ainsi ce désir de retrouver la question du bien vivre. Aujourd'hui, ce ne sont que les prémisses d'un refus déjà très marqué de se soumettre à une politique d'austérité qui ne fait que pousser les individus dans une plus grande pauvreté économique, sociale et politique : celle de l'abdication face à des lois qui semblent échapper à tout contrôle ou limite. Il s'agit d'une quête assez globale qui lie et relie les êtres, aussi hétérogènes et différenciés soient-ils, dans un même soulèvement contre l'inique et pour un projet commun, de bien vivre.

Le troisième trait qui dessine le visage de la démocratie est sans doute le plus difficile, celui sur lequel les Révolutions ont toutes échoué et où le réifié a pu reprendre la main : l'auto-institution de la politique. Refuser l'institution, car dans son déploiement, elle se sépare inlassablement de ce qui la fonde, l'agir démocratique. Car, comme le signale si justement Adorno, l'institution tend à préférer la forme réifiée contre les subjectivités et à imposer la norme contre l'hétérogène ${ }^{12}$. Là sans doute réside la limite à laquelle les contestations s'épuisent et se perdent: cette impossibilité encore jamais dépassée de trouver la manière d'endiguer la réification. Deleuze et Guattari ont donné la réponse et 
l'appui dans le concept d'une révolution toujours en mouvement ${ }^{13}$. En perpétuel mouvement. Mais les mouvements s'épuisent aussi à chercher à maintenir cette instabilité.

Aujourd'hui, les choses bougent. La louve a secoué ses mamelles et poussé un cri. L'espoir se trouve sans aucun doute dans la capacité d'invention des êtres. Miser sur la potentialité des êtres à l'invention, sur leur capacité de trouver une manière de tenir en respect la réification, la ratio, à travers l'essor et la composition d'un monde où l'instable serait au cœur du dispositif démocratique pour protéger la démocratie de ses propres démons. Tel pourrait être l'enjeu exigeant des temps qui s'annoncent.

\section{NOTES}

1. Dans le titre de cet article nous indiquons les auteurs les plus proches qui marquent cette constellation; évidemment, des auteurs comme Aristote, La Boétie, Marx, Nietzsche, Adorno, Lévinas, ou Negt, et bien d'autres participent de cette voûte étoilée. Mais il me semblait important de rendre hommage à des auteurs qui ont porté une critique autre dans un univers intellectuel dominé par l'Alpha et l'Oméga de l'État et de l'économique.

2. Naomi Klein, La stratégie du choc, la montée d'un capitalisme du désastre, Paris, Acte Sud, 2007.

3. Miguel Abensour, La démocratie contre l'État, Marx et le moment machiavélien, Paris, Puf, 1997.

4. Voir la page concernant le Manifeste d'économistes atterrés de 2010 : http://atterres.org/ node/ 4157

5. Jean-Marie Vincent, Fétichisme et société, Paris, Anthropos, 1971.

6. Pierre Clastres, La société contre l'État, Paris, Les éditions de Minuit, 1974.

7. Claude Lefort, L'invention démocratique, Paris, Fayard, 1981.

8. Jacques Rancière, La haine de la démocratie, Paris, Hazan, 2005.

9. Référence au titre du dernier ouvrage de John Holloway, Crack capitalism, Pluto Press, 2010.

10. Stéphane Hessel, Indignez-vous !, Montpellier, Indigène, 2010.

11. Françoise Proust, De la résistance, Les éditions du Cerf, 1997.

12. Theodor W. Adorno, Métaphysique, Paris, Les éditions Payot, 2006.

13. Merci à Anne Querrien d'avoir attiré mon attention sur ce point.

\section{AUTEUR}

\section{LUCIA SAGRADINI}

Chercheuse en sociologie 